\title{
ОСАЖДЕНИЕ НИТРОЗОРЕЗОРЦИНОВЫХ КОМПЛЕКСОВ КОБАЛЬТА С ПОМОЩЬЮ АЛКИЛАМИНОВ
}

\author{
(Представил О. Киррет)
}

Ранее нами было показано, что синтезированные из сланцевого сырья 4-нитрозопроизводные 5-метилрезорцина и 2,5-диметилрезорцина (соответственно MRes-NO и DMRes-NO) имеют $\mathrm{p} K_{a}$ на 3,5-4 единицы меньше, чем нитрозофенол, нитрозонафтолы и нитрозо-R-соль [1]. Благодаря этому пределы $\mathrm{pH}$ комплексообразования их с ионами металлов расширяются в сторону более кислых растворов, где можно ожидать более высокой селективности по отношению к легко гидролизующимся катионам. В слабокислой среде оба нитрозосоединения (АН) образуют с кобальтом комплексное соединение состава $\mathrm{CoA}_{3}$.

Известно множество примеров, когда разнолигандные комплексы образуют с поверхностно-активными лигандами тройные соединения $\left.{ }^{2}\right]$. Это имеет место также в случае нитрозокомплексов, так как комплекс с нитрозо-R-солью хорошо поддается экстракции органическими растворителями, содержащими длинноцепочечные четвертичные соли алкиламмония $\left[{ }^{3,4}\right]$. Нами найдено, что в случае прибавления алкиламинов $(\mathrm{Am})$ к водным растворам комплексов кобальта с нитрозорезорцинами растворы мутнеют и из них выпадает осадок.

Цель настоящей работы - исследование осаждения нитрозорезорцината кобальта алкиламинами в виде тройного комплексного соединения.

\section{Определение состава и произведения растворимости тройного комплекса}

В опытах по установлению состава тройных комплексов кобальт осаждали из его $1 \cdot 10^{-4}$ молярных растворов, варьируя количество нитрозссосдинения и гидрохлорида додециламина в области $(3-8) \cdot 10^{-4}$ моль/дм ${ }^{3}$. Так как нам не удалось найти метода для прямого определения количества амина, связанного в осадок, соотношение амина и $\mathrm{CoA}_{3}$ определяли по разности масс осадка и содержащегося в нем $\mathrm{CoA}_{3}$. Массу последнего определяли по содержанию кобальта в осадке. Для анализа выпавшие осадки отделяли на стеклянном фильтре и высушивали при $105^{\circ} \mathrm{C}$. Взвешенные осадки растворяли в смеси этилового спирта и уксусной кислоты (1:1 по объему), после чего определяли содержание кобальта в полученных растворах на атомном абсорбере AAS-1N (длина волны 240,7 нм, пламя ацетилен-воздух). Однако полученные результаты показали, что вследствие соосаждения реагентов доля кобальта в осадке в некоторой степени зависит от исходного соотношения кобальта и реагентов. Поэтому истинную молекулярную массу тройного комплекса (без соосажденных реагентов) приходилось определять экстраполяцией кажущихся величин молекулярной массы до нулевой концентрации реагентов. Для комплекса MRes-NO получили значение молекулярной массы 1060, для комплекса DMRes-NO - 1120. Это 
дает право приписать обоим тройным комплексам формулу $\mathrm{CoA}_{3} \mathrm{Am}_{3}$ (теор. молекулярные массы, соответственно, 1073 и 1115).

Для определения произведения растворимости $L\left(L \equiv[\mathrm{CoA}][\mathrm{Am}]^{3}\right.$, где $[\Lambda \mathrm{m}]$ - равновесная концентрация амина) тройных комплексов $\mathrm{CoA}_{3} \mathrm{Am}_{3}$, характеризующего полноту их осаждения, была поставлена серия опытов, в которых концентрацию кобальта в исходных растворах варьировали в интервале $(0,6-1,0) \cdot 10^{-4}$ моль/дм ${ }^{3}$. Соотношение исходных концентраций кобальта, нитрозорезорцина и додециламина в опытах поддерживали равным $1: 3: 3$, величину $\mathrm{pH}$ среды - 5,6-5,7 (ацетатный буферный раствор, ионная сила 0,005$)$. На следующий день после прибавления реагентов образовавшийся осадок отфильтровали через плотную бумагу «синяя лента». В фильтрате методом атомной абсорбции определяли суммарную концентрацию кобальта (в виде катиона и комплекса), пропорциональную концентрации несвязанного амина ([Am]/3 ), Концентрацию $\mathrm{CoA}_{3}$ определяли спектрофотометрически при $\lambda=500$ нм, учитывая, что в данном случае вместо [AH] в уравнение (6) в ['] необходимо ввести общую концентрацию нитрозорезорцина в фильтрате, равную равновесной концентрации амина.

Результаты опытов (таблица) показали, что равновесные концентрации компонентов во всех растворах практически постоянны. Получен. ная из этих концентраций величина $L$ для тройного комплекса $\mathrm{CoA}_{3} \mathrm{Am}_{3}$ при $\mathrm{AH}=$ MRes-NO составляет $(0,63 \pm 0,09) \cdot 10^{-16} \quad\left(\text { моль } \cdot \text { дм }^{-3}\right)^{4}, \quad$ при $\mathrm{AH}=\mathrm{DMRes}-\mathrm{NO}-(0,40 \pm 0,03) \cdot 10^{-16}\left(\text { моль } \cdot \text { дм }^{-3}\right)^{4}$.

Концентрация $\left[\mathrm{Co}^{2+}\right]+\left[\mathrm{CoA}_{3}\right],[\mathrm{Am}]$ и $\left[\mathrm{CoA}_{3}\right]$, моль $\cdot$ дм-3.104, в равновесных растворах при различных исходных концентрациях компонентов и произведение растворимости комплексов $\mathrm{CoA}_{3} \mathrm{Am}_{3} L$ (моль $\left.-д м-3\right)^{4} \cdot 10^{16}$, при рН 5,6-5,7

\begin{tabular}{|c|c|c|c|c|c|c|c|c|c|}
\hline \multirow[b]{2}{*}[\mathrm{Co}^{2+}]{$_{0}$} & \multirow[b]{2}{*}{ 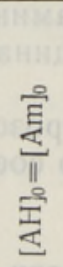 } & \multicolumn{4}{|c|}{ MRes-NO } & \multicolumn{4}{|c|}{ DMRes-NO } \\
\hline & & 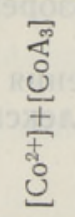 & {$[\mathrm{Am}]$} & {$\left[\mathrm{CoA}_{3}\right]$} & $\sqrt{60}$ & 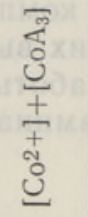 & $\cos (x)$ & {$\left[\mathrm{CoA}_{3}\right]$} & . \\
\hline 0,6 & 1,8 & 0,420 & 1,26 & 0,312 & 0,62 & 0,416 & 1,25 & 0,210 & 0,41 \\
\hline 0,7 & 2,1 & 0,414 & 1,24 & 0,309 & 0,59 & 0,420 & 1,26 & 0,212 & 0,42 \\
\hline 0,8 & 2,4 & 0,432 & 1,30 & 0,328 & 0,72 & 0,413 & 1,24 & 0,203 & 0,39 \\
\hline 0,9 & 2,7 & 0,424 & 1,27 & 0,308 & 0,63 & 0,413 & 1,24 & 0,216 & 0,41 . \\
\hline 1,0 & 3,0 & 0,430 & 1,26 & 0,306 & 0,61 & 0,410 & 1,27 & 0,200 & 0,37 \\
\hline
\end{tabular}

\section{Зависимость степени осаждения кобальта от рН среды и исходных концентраций компонентов}

Количество кобальта, осаждающегося в виде комплекса $\mathrm{CoA}_{3} \mathrm{Am}_{3}$, определяют следующие равновесные процесы:

1. Кислотная диссоциация нитрозосоединения

$$
\mathrm{AH} \rightleftarrows \mathrm{H}^{+}+\mathrm{A}^{-}
$$

с константой ионизации

$$
K_{a}=\frac{\left[\mathrm{H}^{+}\right]\left[\mathrm{A}^{-}\right]}{[\mathrm{AH}]} .
$$


2. Комплексообразование кобальта с ионизированной формой нитрозосоединения $\mathrm{A}^{-}$

$$
\mathrm{Co}^{3+}+3 \mathrm{~A}^{-} \rightleftarrows \mathrm{CoA}_{3}
$$

с константой устойчивости комплекса *

$$
\beta_{3}=\frac{\left[\mathrm{CoA}_{3}\right]}{\left[\mathrm{Co}^{2+}\right]\left[\mathrm{A}^{-}\right]^{3}} .
$$

3. Образование с Am малорастворимсго тройного комплекса $\mathrm{CoA}_{3} \mathrm{Am}_{3}$

$$
\mathrm{CoA}_{3}+3 \mathrm{Am} \rightleftharpoons\left(\mathrm{CoA}_{3} \mathrm{Am}_{3}\right)_{\text {осадок }}
$$

с произведением растворимости

$$
L=\left[\mathrm{CoA}_{3}\right][\mathrm{Am}]^{3} .
$$

Обозначив исходные концентрации кобальта, нитрозосоединения и амина через $\left[\mathrm{Co}^{2+}\right]_{0},[\mathrm{AH}]_{0}$ и $[\mathrm{Am}]_{0}$, можем записать уравнения материального баланса этих компонентов:

$$
\begin{gathered}
{\left[\mathrm{Co}^{2+}\right]_{0}=\left[\mathrm{Co}^{2+}\right]+\left[\mathrm{CoA}_{3}\right]+\left[\mathrm{CoA}_{3} \mathrm{Am}_{3}\right],} \\
{[\mathrm{AH}]_{0}=[\mathrm{AH}]+\left[\mathrm{A}^{-}\right]+3\left[\mathrm{CoA}_{3}\right]+3\left[\mathrm{CoA}_{3} \mathrm{Am}_{3}\right],} \\
{[\mathrm{Am}]_{0}=[\mathrm{Am}]+3\left[\mathrm{CoA}_{3} \mathrm{Am}_{3}\right],}
\end{gathered}
$$

где $\left[\mathrm{CoA}_{3} \mathrm{Am}_{3}\right]$ - количество образовавшегося осадка тройного комплекса, приведенное к единице объема раствора.

Решение системы уравнений (1)-(6) относительно $\left[\mathrm{CoA}_{3} \mathrm{Am}_{3}\right]$ дает степень осаждения кобальта

$$
\alpha=\frac{\left[\mathrm{CoA}_{3} \mathrm{Am}_{3}\right]}{\left[\mathrm{Co}^{2+}\right]_{0}} .
$$

Решение указанной системы уравнений в общем случае невозможно. Тем не менее эта система может быть приведена к виду только с одним неизвестным - $\left[\mathrm{CoA}_{3} \mathrm{Am}_{3}\right]$ :

$$
\frac{\left[\mathrm{CoA}_{3} \mathrm{Am}_{3}\right]}{\left[\mathrm{Co}^{2+}\right]_{0}}=1-\frac{L}{B^{3}\left[\mathrm{Co}^{2+}\right]_{0}}-\frac{B^{6} L\left(K_{a}+\left[\mathrm{H}^{+}\right]\right)^{3}}{K_{a} \beta_{3}\left[\mathrm{Co}^{2+}\right]_{0}\left(B^{3} \mathrm{C}-3 L\right)^{3}},
$$

где

$$
\begin{aligned}
& B=[\mathrm{Am}]_{0}-3\left[\mathrm{CoA}_{3} \mathrm{Am}_{3}\right], \\
& C=[\mathrm{AH}]_{0}-3\left[\mathrm{CoA}_{3} \mathrm{Am}_{3}\right] .
\end{aligned}
$$

Уравнение (8) легко решить относительно $\left[\mathrm{CoA}_{3} \mathrm{Am}_{3}\right]$ методом последовательного приближения на ЭВМ, если известны $K_{a}, \beta_{3}, L,\left[\mathrm{H}^{+}\right]$, $\left[\mathrm{Co}^{2+}\right]_{0},[\mathrm{AH}]_{0}$ и $[\mathrm{Am}]_{0}$.

Из уравнения (8) следует, что при заданных $\left[\mathrm{Co}^{2+}\right]_{0},[\mathrm{AH}]_{0}$ и $[\mathrm{Am}]_{0}$ осаждение происходит только при величине $\mathrm{pH}$, соответствующей условию

$$
\left[\mathrm{H}^{+}\right]<K_{a}\left(D E^{1 / 3}-1\right),
$$

где

$$
\begin{gathered}
D=[\mathrm{Am}]_{0}^{-3}\left([\mathrm{AH}]_{0}[\mathrm{Am}]_{0}^{3}-3 L\right), \\
E=\beta_{3} L^{-1}\left(\left[\mathrm{Co}^{2+}\right]_{0}[\mathrm{Am}]_{0}^{3}-L\right) .
\end{gathered}
$$

\footnotetext{
* Фактически в растворе несвязанного в комплекс $\mathrm{Co}^{3}+$ не существует. Учитывая полное окисление кобальта при комплексообразовании, в формулах вместо [ $\left.\mathrm{Co}^{3+}\right]$ можем использовать $\left[\mathrm{Co}^{2+}\right]$.
} 


\section{Экспериментальная проверка модели процесса осаждения}

Для проверки модели процесса осаждения $\mathrm{CoA}_{3} \mathrm{Am}_{3}$ были проведены опыты, в которых концентрацию кобальта поддерживали $1 \cdot 10^{-4}$ моль/дм ${ }^{3}$, соотношение $\left[\mathrm{Co}^{2+}\right]_{0}:[\mathrm{AH}]_{0}:[\mathrm{Am}]_{0}$, соответственно, $1: 3: 3$ и $1: 5: 5$ и величину $\mathrm{pH}$ варьировали в пределах $2,7-5,4$. В качестве комплексообразователей AH использовали MRes-NO и DMRes-NO, в качестве $\mathrm{Am}$ - додециламин. $\mathrm{pH}$ регулировали ацетатным и хлористоводородным буферными растворами при ионной силе 0,005 . Чтобы уменьшить влияние адсорбции на образовавшийся осадок, последний фильтровали через определенные промежутки времени после прибавления реагентов. Степень осаждения кобальта рассчитывали по анализу фильтрата методом атомной абсорбционной спектроскопии.

Величины $\alpha$, определенные при соотношении кобальта и реагентов $1: 3: 3$ через сутки, а при соотношении $1: 5: 5$ через трое суток после прибавлення реагентов, хорошо ложатся на кривую, рассчитанную по уравнению (8) (рисунок).
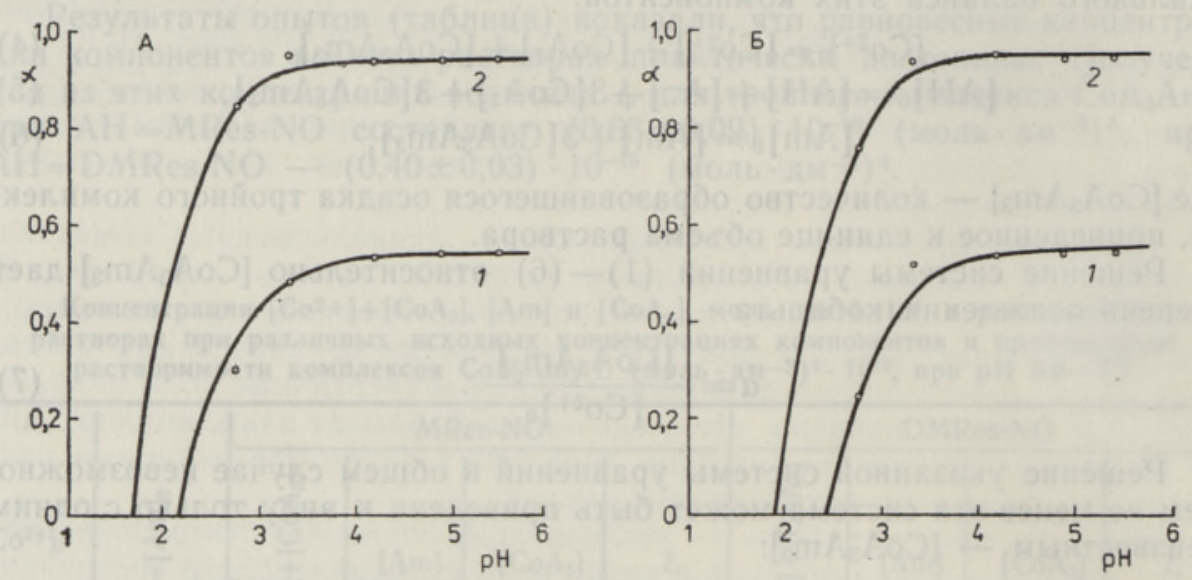

Зависимость степени осаждения кобальта в виде $\mathrm{CoA}_{3} \mathrm{Am}_{3}$ из $1 \cdot 10^{-4}$ молярных растворов от $\mathrm{pH}$ среды. А - комплексы MRes-NO, Б - комплексы DMRes-NO. 1 - $\left[\mathrm{Co}^{2}+\right]_{0}$ : $[\mathrm{AH}]_{0}:[\mathrm{Am}]_{0}=1: 3: 3 ; 2-\left[\mathrm{Co}^{2+}\right]_{0}:[\mathrm{AH}]_{0}:[\mathrm{Am}]_{0}=1: 5: 5$.

Расчетные кривые получали с использованием величин $L$ из настоящей работы и значений $K_{a}$ и $\beta_{3}$ из [ [ $\left.{ }^{1}\right]$ : для MRes-NO 4,90 $\cdot 10^{-4}$ и $18,1 \cdot 10^{12}$, для DMRes-NO $3,47 \cdot 10^{-4}$ и $3,83 \cdot 10^{12}$.

Таким образом, в случае равновесного процесса экспериментальные данные подтверждают правильность теоретической модели.

Однако путем определения кобальта в остаточном растворе в течение длительного периода после прибавления реагентов обнаружилось, что указанное согласие сохраняется более или менее четко только в первые трое суток. Затем происходит медленное, но постоянное накопление осадка. Этот процесс наблюдается даже спустя месяц после начала реакции. Скорость образования тройного комплекса кобальта с нитрозорезорцинами и алкиламинами - предмет нашего дальнейшего изучения.

\section{Выводы}

1. Установлено, что при введении в раствор нитрозорезорциновых комплексов $\mathrm{CoA}_{3}$ длинноцепочечных алкиламинов Am в осадок выпадает тройное комплексное соединение состава $\mathrm{CoA}_{3} \mathrm{Am}_{3}$. Определено про- 
изведение растворимости этих соединений качестве Am додециламина.

2. Получено уравнение, описывающее зависимость степени осаждения кобальта в виде $\mathrm{CoA}_{3} \mathrm{Am}_{3}$ от $\mathrm{pH}$ среды и исходных концентраций компонентов. Показано его согласие с экспериментальными данными в случае равновесного процесса. Предлагается метод решения неявной формы уравнения методом последовательного приближения.

\section{ЛИТЕ РАТ УРА}

1. Ноханнес И., Мельдер Л. Синтез нитрозорезорцинов и их комплексообразование с кобальтом. - Изв. АН ЭССР. Хим., 1988, 37, № 2, 112-117.

2. Тананайко М. М., Тодрадзе Г. А., Горенитейн Л. И. Влияние длинноцепочечных поверхностно-активных веществ на разнолигандные комплексы. - Ж. анал. хим., 1984, 39, № 6, 1034-1039.

3. Ягодин Г. А., Кузьмин В. И., Федотов О. Н. и др. Способ извлечения кобальта из водных растворов. - Открытия, изобретения, 1982, № 32, авт. свид. СССР № 954471 .

4. Тананайко М. М., Тодрадзе Г. А., Стецюра H. А. Реакция кобальта(II) с нитрозо-Rсолью н хлоридом цетилпиридиния. - Укр. хим. ж., 1986, 52, № 6, 629-633.

Институт химии
Академии наук Эстонской ССР

Поступила в редакцию $3 /$ XII 1987

Ille JOHANNES, L. MOLDER

\section{KOOBALTI JA NITROSORESORTSIINIDE VAHELISTE KOMPLEKSIDE SADESTAMINE ALKUOLAMIINIDEGA}

On näidatud, et nōrgalt happelistest lahustest, mis sisaldavad koobaltit, 4-nitroso-5metüülresortsiini vôi 4-nitroso-2,5-dimetüülresortsiini ja alküülamiini, sadeneb kolmikkompleksühend $\mathrm{CoA}_{3} \mathrm{Am}_{3}$. On määratud ühendite $\mathrm{CoA}_{3} \mathrm{Am}_{3}$ lahustuvuskorrutised, kui $\mathrm{Am}$ on dodetsüülamiin. On saadud võrrand, mis kirjeldab koobalti sadenemisastme sōltuvust lahuse $\mathrm{pH}$-st ja komponentide lähtekontsentratsioonist. Vōrrandi järgi arvutatud tulemused langevad hästi kokku katseandmetega.

\section{Ille JOHANNES, L. MOLDER}

\section{PRECIPITATION OF NITROSORESORCINOL COMPLEXES OF COBALT BY ALKYLAMINES}

It has been ascertained that from slightly acid solutions containing cobalt, 4-nitrose: 5-methylresorcinol or 4-nitroso-2,5-dimethylresorcinol and alkylamine, $\mathrm{CoA}_{3} \mathrm{Am}_{3}$ precipitates. By using dodecylamine the solubility products of the precipitate have been determined.

An equation of the dependence of the degree of cobalt precipitation on the initial concentration of the components and the acidity of solution is presented. Results obtained by the equation are in good accordancẹ with the experimențțal dața, 